\title{
ON QUANTUM AND CLASSICAL POISSON ALGEBRAS
}

\author{
JANUSZ GRABOWSKI \\ Institute of Mathematics, Polish Academy of Sciences \\ Śniadeckich 8, P.O. Box 21, 00-956 Warszawa, Poland \\ E-mail: jagrab@impan.gov.pl \\ NORBERT PONCIN \\ Institute of Mathematics, University of Luxembourg \\ Avenue de la Fä̈encerie, 162A, L-1511 Luxembourg City, Grand-Duchy of Luxembourg \\ E-mail: norbert.poncin@uni.lu
}

\begin{abstract}
Results on derivations and automorphisms of some quantum and classical Poisson algebras, as well as characterizations of manifolds by the Lie structure of such algebras, are revisited and extended. We prove in particular a somewhat unexpected fact that the algebras of linear differential operators acting on smooth sections of two real vector bundles of rank 1 are isomorphic as Lie algebras if and only if the base manifolds are diffeomorphic, whether or not the line bundles themselves are isomorphic.
\end{abstract}

1. Introduction. Let us start with an overview of relevant literature on isomorphisms and derivations of infinite-dimensional Lie algebras.

In [28], Pursell and Shanks proved the well-known result stating that the Lie algebra of all smooth compactly supported vector fields of a smooth manifold characterizes the differentiable structure of the variety. Similar upshots were obtained in numerous subsequent papers dealing with different Lie algebras of vector fields and related algebras (see e.g. $[1,2,3,10,14,19,27,29])$.

2000 Mathematics Subject Classification: Primary 17B63; Secondary 13N10, 16S32, 17B40, 17B65, 53D17.

Key words and phrases: line bundles, differential operators, derivations, automorphisms, Lie algebras, Chevalley cohomology, one-parameter groups.

Research of Janusz Grabowski was supported by the Polish Ministry of Scientific Research and Information Technology under grant No. 2 P03A 02024. Research of Norbert Poncin was supported by grant R1F105L10.

The paper is in final form and no version of it will be published elsewhere. 
Derivations of certain infinite-dimensional Lie algebras arising in Geometry were also studied in different situations (note that in infinite dimensions there is no such clear correspondence between derivations and one-parameter groups of automorphisms as in the finite-dimensional case). Let us mention a result of L. S. Wollenberg [31] who described all derivations of the Lie algebra of polynomial functions on the canonical symplectic space $\mathbb{R}^{2}$ with respect to the Poisson bracket. It turned out that there are outer derivations of this algebra in contrast to the corresponding Weyl algebra. This can be viewed as a variant of a "no-go" theorem (see [20]) stating that the Dirac quantization problem [7] cannot be solved satisfactorily because the classical and the corresponding quantum algebras are not isomorphic as Lie algebras. An algebraic generalization of the latter fact, known as the algebraic "no-go" theorem, has been proved in [9] by different methods. Derivations of the Poisson bracket of all smooth functions on a symplectic manifold have been determined in [4] (for the real-analytic case, see [12]). Another important result is the one by $\mathrm{F}$. Takens [30] stating that all derivations of the Lie algebra $\mathcal{X}(M)$ of smooth vector fields on a manifold $M$ are inner. The same turned out to be valid for analytic cases [11]. Some cases of the Lie algebras of vector fields associated with different geometric structures were studied in a series of papers by Y. Kanie [21]-[24].

Our work [15] contains Shanks-Pursell type results for the Lie algebra $\mathcal{D}(M)$ of all linear differential operators of a smooth manifold $M$, for its Lie subalgebra $\mathcal{D}^{1}(M)$ of all linear first-order differential operators of $M$, and for the Poisson algebra $\mathcal{S}(M)=\operatorname{Pol}\left(T^{*} M\right)$ of all polynomial functions on $T^{*} M$, the symbols of the operators in $\mathcal{D}(M)$. Furthermore, we computed all the automorphisms of these algebras and showed that the Lie algebras $\mathcal{D}(M)$ and $\mathcal{S}(M)$ are not integrable. The paper [17] provides their derivations, so it is a natural continuation of that previous work and can be considered as a generalization of the results of Wollenberg and Takens. It is also shown which derivations generate one-parameter groups of automorphisms and the explicit form of such one-parameter groups is given.

The first part of the present text is of expository nature and contains an intuitive description of the major facts explained in [17]. Moreover, experience of different approaches to the quantization problem and the geometric study of differential equations incite to substitute differential operators acting on tensor densities for differential operators on functions. In the frame of our previous works, this substitution requires investigations on a possible characterization of a manifold $M$ by the Lie algebra of differential operators acting on densities on $M$ of arbitrary fixed weight, or more generally, on the potential characterization, by the canonical Lie algebra structure of the space of linear differential operators on smooth sections of an arbitrary $\mathbb{R}$-line bundle $L$, of the base manifold $M$ or even of the bundle $L$ itself. These problems are solved in the second part of this paper where some new results are proven. Note finally that throughout this paper all manifolds are assumed to be Hausdorff and second countable.

\section{Derivations of some quantum and classical Poisson algebras}

2.1. Locality and weight. In this section we depict the derivations of the algebras $\mathcal{D}^{1}(M)$, $\mathcal{S}(M)$, and $\mathcal{D}(M)$. Let $(\mathcal{D},[.,]$.$) be one of these three filtered Lie algebras and let C$ be a 
derivation of $(\mathcal{D},[.,]$.$) . We speak of operators when referring to elements of \mathcal{D}$ and denote the algebra $C^{\infty}(M)$ of smooth functions of $M$ by $\mathcal{A}$. The adjoint action of a smooth function $f$ of $M$, regarded as a differential operator of order 0 , on an operator $D \in \mathcal{D}^{i}$ lowers the filtration degree by 1 . This provides a tool for proofs by induction. The idea is really fruitful if derivations have weight 0 . Indeed, a bracket such as $[C D, f]$, which involves the chosen derivation, is then also a member of $\mathcal{D}^{i-1}$.

We first prove that any derivation $C$ has a bounded weight, i.e. that there is a positive integer $d$, such that $C \mathcal{D}^{i} \subset \mathcal{D}^{i+d}, \forall i \in \mathbb{N}$. The proof uses the derivation property on functions, the characterization of filters "à la Vinogradov", and the result that the $\mathcal{A}$ module $\Omega^{1}(M)$ of differential 1-forms is spanned by the differentials of a finite number of functions. This last upshot is a consequence of Whitney's embedding theorem.

In order to verify that investigation by local computations is possible, we have to check if any derivation can be restricted to a domain of local coordinates. This means that we must prove that a derivation is always a local operator. We obtain locality using a general technique worked out by De Wilde and Lecomte, see [5]: if an operator $D \in \mathcal{D}$ vanishes in a neighborhood $U$ of a point $x \in M$, it reads $D=\sum_{k}\left[X_{k}, D_{k}\right]$, where the sum is finite and the vector fields $X_{k}$ and operators $D_{k}$ vanish in some neighborhood $V \subset U$ of $x$. The derivation property $C D=\sum_{k}\left(\left[C X_{k}, D_{k}\right]+\left[X_{k}, C D_{k}\right]\right)$ then allows one to conclude.

Let us emphasize that significant information on automorphisms and derivations is encoded in the automorphism and derivation properties written for two functions. If $\left(x^{1}, \ldots, x^{n}\right)$ are local coordinates in an open subset $U \subset M$, we get

$$
0=C\left[x^{i}, x^{j}\right]=\left[C x^{i}, x^{j}\right]+\left[x^{i}, C x^{j}\right] .
$$

The values $C x^{i}$ are differential operators over $U$ or polynomials of $T^{*} U$. In the first case, we symbolically write the derivatives in these operators $C x^{i}$ as monomials in the corresponding components $\left(\xi_{1}, \ldots, \xi_{n}\right)$ of some linear form $\xi \in\left(\mathbb{R}^{n}\right)^{*}$. Hence $C x^{i} \simeq P^{i}$, where the $P^{i}$ are polynomials of $T^{*} U$. In this polynomial language, the above result reads

$$
\partial_{\xi_{j}} P^{i}=\partial_{\xi_{i}} P^{j}
$$

Integration furnishes us with a polynomial $P$ such that $\partial_{\xi_{i}} P=P^{i}$, i.e. $\left[P, x^{i}\right]=C x^{i}$. So derivation $C$ coincides on coordinate functions with an interior derivation. It is easily seen that for an arbitrary function $f$, the derivations $C$ and ad $P$ differ by a function, $C f-[P, f] \in \mathcal{A}$, i.e. locally $C-$ ad $P$ respects the lowest filter. After a gluing process and a generalization to higher order filters, we conclude that any derivation can be corrected by an interior derivation in such a way that the filtration is respected. We will refer to this property as "property P1". Let us stress that $P \in \mathcal{D}^{d+1}$ since the weight of $C$ is $d$. The operator $P$ is not unique, the set of all convenient $P$ is $P+\mathcal{D}^{1}$.

2.2. Restriction to functions. We prove that for any derivation $C$ that respects the filtration there is a unique vector field $Y$ such that the derivation $C-\operatorname{ad} Y$ respects the filtration and reduces on functions to a multiple of identity, i.e. $\left.(C-\operatorname{ad} Y)\right|_{\mathcal{A}}=\kappa$ id, where $\kappa \in \mathbb{R}$ is uniquely determined by $C$. 
In the following we refer to this result as "property P2". Unless differently stated, we assume that all derivations examined below have been corrected and have acquired properties $\mathrm{P} 1$ and $\mathrm{P} 2$.

The proof of the preceding upshot is based upon a technique similar to that used in [16, Sect. 2.3.2] and will not be described here.

2.3. Derivations of first order linear differential operators. The Lie algebra of first order differential operators has a canonical splitting, $\mathcal{D}^{1}(M)=\mathcal{A} \oplus \mathcal{X}(M)$, where $\mathcal{X}(M)$ is the Lie algebra of vector fields of $M$. In the following we simply write $\mathcal{D}^{1}$ and $\mathcal{X}$, if no misunderstanding is possible. In view of property $\mathrm{P} 2$, we have $C f=\kappa f(f \in \mathcal{A}, \kappa \in \mathbb{R})$. The derivation property shows that $\left.C\right|_{\mathcal{X}}$ is a 1-cocycle of the canonical representation of the Lie algebra $\mathcal{X}$ on the space $\mathcal{A}$. These cocycles are well-known (see [8], [6]): $C X=$ $\lambda \operatorname{div} X+\omega(X)\left(X \in \mathcal{X}, \lambda \in \mathbb{R}, \omega \in \Omega^{1}(M) \cap\right.$ ker d). So we know $C$ on any first-order operator. If we wish to recover the initial, not yet corrected (see 2.2) derivation (we denote it also by $C$ ), we have to add again the corrections. Finally,

$$
C(f+X)=[Y, f+X]+\kappa f+\lambda \operatorname{div} X+\omega(X), \quad \forall f \in \mathcal{A}, \forall X \in \mathcal{X},
$$

where $Y \in \mathcal{X}, \kappa, \lambda \in \mathbb{R}, \omega \in \Omega^{1}(M) \cap$ kerd are uniquely defined by $C$. The cohomological translation of this result is

$$
H^{1}\left(\mathcal{D}^{1}, \mathcal{D}^{1}\right)=\mathbb{R}^{2} \oplus H_{D R}^{1}(M) .
$$

Here $H^{1}\left(\mathcal{D}^{1}, \mathcal{D}^{1}\right)$ is the first cohomology space of the Lie algebra $\mathcal{D}^{1}$ and $H_{D R}^{1}(M)$ is the first de Rham cohomology group of the underlying manifold $M$.

Some explanations regarding divergences can be found for instance in [26]. Let us recall that any nowhere vanishing 1-density $\rho_{0}$ defines a vector space isomorphism $\tau_{0}$ between the space of 1-densities and the space of functions. Nevertheless these spaces are not isomorphic as modules over the Lie algebra of vector fields. Indeed, if $\mathcal{L}_{X}$ and $L_{X}$ denote the Lie derivatives with respect to a vector field $X$, of 1-densities and functions respectively, the difference $\tau_{0} \circ \mathcal{L}_{X} \circ \tau_{0}^{-1}-L_{X}$ is the value at $X$ of a 1-cocycle of $\mathcal{X}$ with coefficients in $\mathcal{A}$. This cocycle is the divergence implemented by $\rho_{0}$. There is no canonical divergence. Nevertheless all divergences induced by nowhere vanishing 1-densities are cohomologous. So these divergences define a privileged cohomology class. The divergence above and below is a fixed divergence of this class. An approach to divergences for general Lie algebroids can be found in [18].

2.4. Derivations of polynomials on the cotangent bundle. Any (corrected) derivation $C$ of $\mathcal{S}(M)=\operatorname{Pol}\left(T^{*} M\right)$ (in the following we simply write $\mathcal{S}$ ) restricts to a derivation (still denoted by $C$ ) of the Lie algebra $\mathcal{S}^{1}$ of polynomials of degree 1 at most. Since this algebra is isomorphic to $\mathcal{D}^{1}$, the derivation $C$ reads

$$
C(f+X)=\kappa f+\lambda \operatorname{div} X+\omega(X), \quad \forall f \in \mathcal{A}, \forall X \in \mathcal{X} .
$$

If we impose the derivation condition, not only for elements of $\mathcal{S}^{1}$ but for all polynomials in $\mathcal{S}$, the terms of the r.h.s. of Equation (1) either cancel or turn out to be the traces on the $\mathcal{S}^{1}$-level of derivations of the whole algebra $\mathcal{S}$. In this intuitive approach we confine ourselves to trying to extend these terms as derivations of $\mathcal{S}$. 
Since $\mathcal{S}$ is a graded algebra, one of its derivations is the so-called degree derivation,

$$
\text { Deg : } \mathcal{S}_{i} \ni P \rightarrow(i-1) P \in \mathcal{S}_{i},
$$

which just multiplies by the (shifted) degree of the argument. Of course $\mathcal{S}_{i}$ is the space $\operatorname{Pol}^{i}\left(T^{*} M\right)$ of homogeneous polynomials of degree $i$. Visibly $-\kappa$ Deg is a derivation of $\mathcal{S}$ that extends the first term of the r.h.s. of Equation (1).

It should be clear that such an extension does not exist for the second term $\lambda$ div.

Since $\omega$ is locally exact, its value at $X$ locally reads

$$
\omega(X)=(\mathrm{d} f)(X)=\{X, f\},
$$

where $f$ is a local function and where $\{.,$.$\} is the standard Poisson bracket of T^{*} M$. So, if, for any polynomial $P \in \mathcal{S}$, we locally define

$$
\bar{\omega}(P):=\{P, f\}=\Lambda(\mathrm{d} P, \mathrm{~d} f),
$$

where $\Lambda$ is the corresponding Poisson tensor, we see that $\bar{\omega}$ is a well and globally defined derivation of $\mathcal{S}$ that extends our third term. It is obvious from the preceding equation that $\bar{\omega}$ is the (vertical) vector field $\omega^{v}$ of $T^{*} M$ induced by $\omega$.

Finally we understand that any derivation $C$ of $\mathcal{S}$ is of the type

$$
C(P)=\{Q, P\}-\kappa \operatorname{Deg} P+\omega^{v}(P), \quad \forall P \in \mathcal{S},
$$

where $Q \in \mathcal{S}, \kappa \in \mathbb{R}, \omega \in \Omega^{1}(M) \cap$ ker d. Let us still mention that $\kappa$ is unique, whereas the set of appropriate $(Q, \omega)$ is $\{(Q+h, \omega+\mathrm{d} h), h \in \mathcal{A}\}$. The cohomological version of this second result is

$$
H^{1}(\mathcal{S}, \mathcal{S})=\mathbb{R} \oplus H_{\mathrm{DR}}^{1}(M),
$$

with self-explaining notations.

2.5. Derivations of linear differential operators. The intuitive approach is as in Section 2.4, conclusions are similar. Note nevertheless that the degree derivation, which extends the first term of Equation (1), is tightly connected with the grading of the classical Poisson algebra $\mathcal{S}$. Since the quantum algebra $\mathcal{D}(M)(\mathcal{D}$ for short) is only filtered, we guess that such an extension is no longer possible on the quantum level.

We now understand that any derivation $C$ of $\mathcal{D}$ has the form

$$
C(D)=[\Delta, D]+\bar{\omega}(D), \quad \forall D \in \mathcal{D},
$$

where $\Delta \in \mathcal{D}, \omega \in \Omega^{1}(M) \cap$ ker d. The lowering (its weight with respect to the filtration degree is -1$)$ derivation $\bar{\omega}$ is defined as in Section 2.4. The convenient $(\Delta, \omega)$ are again $\{(\Delta+h, \omega+\mathrm{d} h), h \in \mathcal{A}\}$. Moreover,

$$
H^{1}(\mathcal{D}, \mathcal{D})=H_{\mathrm{DR}}^{1}(M) .
$$

3. Integrability of derivations. Let us come back to the derivations of the algebras $\mathcal{D}^{1}(M), \mathcal{S}(M)$, and $\mathcal{D}(M)$. For these non-integrable infinite-dimensional Lie algebras, there is no such clear correspondence between derivations and 1-parameter groups of automorphisms as in the finite-dimensional setting. Our goal is to find in each of these cases the most general form of a 1-parameter group of automorphisms. Moreover, computations should unmask a derivation that can be viewed as the generator of the chosen group of 
automorphisms. Finally, we wonder if it is possible to characterize those derivations that induce 1-parameter groups of automorphisms.

First remark that any diffeomorphism $\phi$ of $M$ canonically induces an automorphism $\phi_{*}$ of the considered algebra $\mathcal{D}$. If $\mathcal{D}=\mathcal{D}^{1}(M)$ or $\mathcal{D}=\mathcal{D}(M)$, this automorphism is defined by

$$
\left(\phi_{*} D\right) f=D(f \circ \phi) \circ \phi^{-1}, \quad \forall D \in \mathcal{D}, \forall f \in \mathcal{A} .
$$

If $\mathcal{D}=\mathcal{S}(M)=\operatorname{Pol}\left(T^{*} M\right)$, we set

$$
\phi_{*} P=P \circ\left(\phi^{\sharp}\right)^{-1}, \quad \forall P \in \mathcal{D},
$$

where $\phi^{\sharp}$ is the phase lift of $\phi$. So 1-parameter groups of diffeomorphisms are special 1parameter groups of automorphisms, known a priori, since they are just flows of complete vector fields. Note that there are diffeomorphisms, arbitrarily close to identity, which cannot be embedded in flows $[13,25]$.

We have shown in [15] that any automorphism $\Phi$ of $\mathcal{D}^{1}(M)$ has the form

$$
\Phi(f+X)=\phi_{*}(X)+(K f+\Lambda \operatorname{div} X+\Omega(X)) \circ \phi^{-1}, \quad \forall f \in \mathcal{A}, \forall X \in \mathcal{X},
$$

where $\phi \in \operatorname{Diff}(M), K \in \mathbb{R} \backslash\{0\}, \Lambda \in \mathbb{R}$, and $\Omega \in \Omega^{1}(M) \cap$ ker d are uniquely determined by the chosen automorphism. Let $\Phi_{t}=\Phi_{\phi_{t}, K_{t}, \Lambda_{t}, \Omega_{t}}$ be an arbitrary 1-parameter group of automorphisms. Smoothness with respect to the differential structure of $M$ is assumed. In other words, we suppose that the map

$$
\mathbb{R} \times M \ni(t, x) \rightarrow\left(\Phi_{t} D\right)(f)(x) \in \mathbb{R}
$$

is smooth for any $D \in \mathcal{D}^{1}(M)$ and any $f \in \mathcal{A}$. When computing the l.h.s. of the group condition

$$
\Phi_{\phi_{t}, K_{t}, \Lambda_{t}, \Omega_{t}} \circ \Phi_{\phi_{s}, K_{s}, \Lambda_{s}, \Omega_{s}}=\Phi_{\phi_{t+s}, K_{t+s}, \Lambda_{t+s}, \Omega_{t+s}},
$$

we get terms that can easily be compared with the corresponding terms of the r.h.s., except for one term,

$$
\Lambda_{t}\left(\operatorname{div} \phi_{s *} X\right) \circ \phi_{t}^{-1}
$$

which is not of one of the four types in the r.h.s. of Equation (2). So this term has to be transformed.

Let us recall that the divergence is implemented by a fixed nowhere vanishing 1-density $\rho_{0}$. It is quite obvious that the divergence of the push-forward of a vector field $X$ coincides with the divergence with respect to the pull-back of $\rho_{0}$. More precisely,

$$
\operatorname{div}_{\rho_{0}} \phi_{*} X=\left(\operatorname{div}_{\phi^{*} \rho_{0}} X\right) \circ \phi^{-1},
$$

where the subscript $s$ has been omitted. It is clear that for any diffeomorphism $\phi$ there is a unique positive smooth function $J(\phi)$, such that

$$
\phi^{*} \rho_{0}=(J(\phi)) \rho_{0} .
$$

Furthermore, the reader might have guessed that the essential local building block of $J(\phi)(x)$ is $\left|\operatorname{det} \partial_{x} \varphi\right|$, where $\varphi$ is the local form of $\phi$. Hence, the following property of $J$ :

$$
J(\phi \circ \psi)=\psi^{*}(J(\phi)) . J(\psi), \quad \forall \phi, \psi \in \operatorname{Diff}(M) .
$$


Recall now that if $G$ is a group and $A$ is a left $G$-module, a group 1-cocycle is a map $C: G \rightarrow A$ such that

$$
C\left(g_{1} \cdot g_{2}\right)=g_{1} \cdot\left(C\left(g_{2}\right)\right)+C\left(g_{1}\right), \quad \forall g_{1}, g_{2} \in G,
$$

where "." is the action of $G$ and "." the group multiplication. Note that this cocyclecondition is similar to that of the Hochschild cohomology of an associative algebra. The unique difference between Equation (7) and Equation (8) is the operation in the r.h.s. When applying the logarithm to both sides of Equation (7), we finally get

$$
(\ln \circ J)(\phi \circ \psi)=\psi^{*}((\ln \circ J)(\phi))+(\ln \circ J)(\psi) \text {. }
$$

So

$$
\ln \circ J \in \mathcal{Z}^{1}\left(\operatorname{Diff}(M), C^{\infty}(M)\right)
$$

is a 1-cocycle of the group of diffeomorphisms valued in the module of smooth functions. Moreover, it can be proven that

$$
(\ln \circ J)(\operatorname{Exp}(t X))=\int_{0}^{t} \operatorname{div} X \circ \operatorname{Exp}(s X) \mathrm{ds},
$$

for any complete vector field $X$. Equations (6), (9), and (10) show that

$$
\operatorname{Div}=\ln \circ J
$$

is the group analogue of divergence.

This group divergence allows us to rewrite term (4) in an appropriate form. Starting from Equation (5), we obtain

$$
\begin{aligned}
\left(\operatorname{div}_{\phi^{*} \rho_{0}} X\right) \circ \phi^{-1} & =\left(\operatorname{div}_{(J(\phi)) \rho_{0}} X\right) \circ \phi^{-1} \\
& =\left(\operatorname{div}_{\rho_{0}} X+X((\ln \circ J)(\phi))\right) \circ \phi^{-1} \\
& =(\operatorname{div} X+\mathrm{d}(\operatorname{Div} \phi)(X)) \circ \phi^{-1} .
\end{aligned}
$$

The terms of group condition (3) can now easily be compared. This comparison leads to the equations

$$
\begin{gathered}
\phi_{t} \circ \phi_{s}=\phi_{t+s}, \quad \phi_{0}=\mathrm{id} \\
K_{t} K_{s}=K_{t+s}, \quad K_{0}=1, \\
\Lambda_{t}+K_{t} \Lambda_{s}, \quad \Lambda_{0}=0, \\
K_{t} \Omega_{s}+\phi_{s}^{*} \Omega_{t}+\Lambda_{t} \mathrm{~d}\left(\operatorname{Div} \phi_{s}\right)=\Omega_{t+s}, \quad \Omega_{0}=0,
\end{gathered}
$$

where id is the identity map. The first of these results for instance means that the 1parameter family of diffeomorphisms is actually a 1-parameter group of diffeomorphisms, i.e. the flow of a complete vector field $Y: \phi_{t}=\operatorname{Exp}(t Y)$. Other equations are a little more complicated, but can be solved, so that the explicit form of $K_{t}, \Lambda_{t}$, and $\Omega_{t}$, i.e. of $\Phi_{\phi_{t}, K_{t}, \Lambda_{t}, \Omega_{t}}$ is known. Furthermore, the solutions of the preceding equations involve, in addition to the vector field $Y$, two real numbers $\kappa, \lambda$ and a closed 1-form $\omega$. All these objects are uniquely determined by the chosen 1-parameter group of automorphisms and characterize, as explained in Subsection 2.3, a derivation of $\mathcal{D}^{1}(M)$. We say that this derivation, which is special in the sense that it is associated with a complete vector field, induces the 1-parameter group of automorphisms.

We are now ready to understand the following 
THEOREM 1. A derivation

$$
C_{Y, \kappa, \lambda, \omega}(X+f)=[Y, X+f]+\kappa f+\lambda \operatorname{div} X+\omega(X)
$$

of $\mathcal{D}^{1}(M)$ induces a one-parameter group $\Phi_{t}$ of automorphisms of $\mathcal{D}^{1}(M)$ if and only if the vector field $Y$ is complete. In this case the group is of the form

$$
\begin{aligned}
& \Phi_{t}(X+f)=(\operatorname{Exp}(t Y))_{*}(X)+\left(e^{\kappa t} f+\lambda \frac{e^{\kappa t}-1}{\kappa} \operatorname{div} X\right) \circ \operatorname{Exp}(-t Y) \\
& \quad+\left(\int_{0}^{t} e^{\kappa(t-s)}\left(\lambda \int_{0}^{s} X(\operatorname{div} Y \circ \operatorname{Exp}(u Y)) d u+\left((\operatorname{Exp}(s Y))^{*} \omega\right)(X)\right) d s\right) \circ \operatorname{Exp}(-t Y) .
\end{aligned}
$$

Similar upshots have been obtained for the algebras $\mathcal{S}(M)$ and $\mathcal{D}(M)$. They will not be described here.

4. Differential operators on real line bundles. In [15] we have taken an interest in characterizations of manifold structures, especially by the Lie algebra of linear differential operators acting on the functions of the chosen manifold $M$. We now extend these results to the Lie algebra of differential operators acting on tensor densities over $M$ of arbitrary weight and even to differential operators acting on the smooth sections of an arbitrary $\mathbb{R}$-line bundle $L$. Our objectives are to examine if this Lie algebra structure recognizes the base manifold $M$ and maybe even the bundle $L$ itself.

Let $\pi: L \rightarrow M$ be a real vector bundle of rank 1 over a smooth, Hausdorff, second countable, and connected manifold. We define the algebra $\mathcal{D}(L)=\cup_{k \in \mathbb{N}} \mathcal{D}^{k}(L)$ of differential operators on $L$ in the standard way. Note first that the space $\operatorname{Sec}(L)$ of smooth sections of $L$ is an $\mathcal{A}$-module, so that any function $f \in \mathcal{A}$ induces an endomorphism $m_{f}: \operatorname{Sec}(L) \ni s \rightarrow f s \in \operatorname{Sec}(L)$ of the space $\operatorname{Sec}(L)$. Then set

$$
\begin{gathered}
\mathcal{D}^{0}(L)=\left\{D \in \operatorname{End}(\operatorname{Sec}(L)):\left[D, m_{f}\right]=0, \forall f \in \mathcal{A}\right\}, \\
\mathcal{D}^{k+1}(L)=\left\{D \in \operatorname{End}(\operatorname{Sec}(L)):\left[D, m_{f}\right] \in \mathcal{D}^{k}(L), \forall f \in \mathcal{A}\right\} \quad(k \in \mathbb{N}),
\end{gathered}
$$

where $\operatorname{End}(\operatorname{Sec}(L))$ denotes the algebra of endomorphisms of $\operatorname{Sec}(L)$ and [., .]- the commutator bracket associated with the composition multiplication.

Proposition 1. Any differential operator on $L$ is a local operator.

Proof. Indeed, if we denote $\mathcal{D}^{-1}(L)=\{0\}$, then we can proceed now by induction and consider a $k$ th-order $(k \geq 0)$ differential operator $D$ on $L$, a section $s \in \operatorname{Sec}(L)$ that vanishes in an open subset $U \subset M$, an arbitrary point $x \in U$, and a function $\alpha \in \mathcal{A}$, which vanishes outside $U$ and has constant value 1 in some neighborhood of $x$ in $U$. Since $\left[D, m_{\alpha}\right] \in \mathcal{D}^{k-1}(L)$ is a local operator, $D(\alpha s)=\alpha D(s)$ in $U$, so $(D(s))(x)=D(0)=0$.

REMARK 1. In the following we write $\mathcal{D}(L)$ in the form $\mathcal{D}(L \rightarrow M)$, if we wish to put emphasis on the base manifold $M$, and in the form $\mathcal{D}(M)$, if $L$ is the trivial bundle $M \times \mathbb{R}$. This algebra $\mathcal{D}(M)$ is nothing but the usual algebra of linear differential operators acting on the space of smooth functions of $M$.

Proposition 2. The space $\mathcal{D}(L)=\cup_{k \in \mathbb{N}} \mathcal{D}^{k}(L)$ is a quantum Poisson algebra in the sense of [15]. 
Proof. In view of the above proposition it is sufficient to check it locally. Let $U$ be an open subset of $M$ such that $L$ is trivial over $U$. Any local trivialization of $L$ in $U$, i.e. any nowhere vanishing section $\varsigma \in \operatorname{Sec}\left(L_{U}\right)$ of $L$ over $U$, induces a canonical vector space isomorphism

$$
\iota_{\varsigma}: \operatorname{Sec}\left(L_{U}\right) \ni \varphi \varsigma \rightarrow \varphi \in C^{\infty}(U) .
$$

This isomorphism induces itself an isomorphism of quantum Poisson algebras,

$$
\mathcal{I}_{\varsigma}: \mathcal{D}\left(L_{U}\right) \ni \Delta \rightarrow \iota_{\varsigma} \circ \Delta \circ \iota_{\varsigma}^{-1} \in \mathcal{D}(U) .
$$

A straightforward induction on the degree of differentiation allows one to see that $\mathcal{I}_{\varsigma}$ respects the filtration.

A gauge change entails a change in the identification of $\mathcal{D}\left(L_{U}\right)$ with $\mathcal{D}(U)$. Indeed, if $\varsigma^{\prime}$ is another nowhere vanishing section of $L_{U}$, we have $\varsigma^{\prime}=\psi \varsigma, \psi \in C^{\infty}(U)$, and, as easily verified,

$$
\mathcal{I}_{\varsigma}(\Delta)=m_{\psi} \circ \mathcal{I}_{\varsigma^{\prime}}(\Delta) \circ m_{\psi^{-1}}, \quad \forall \Delta \in \mathcal{D}\left(L_{U}\right),
$$

so that $\mathcal{I}_{\varsigma^{\prime}}^{-1} \circ \mathcal{I}_{\varsigma}$ is a Lie algebra automorphism of $\mathcal{D}\left(L_{U}\right)$. Hence the local isomorphisms $\mathcal{I}_{\varsigma}$ cannot be glued canonically. Note nevertheless that if $\psi$ is a (non-zero) constant, the identifications $\mathcal{I}_{\varsigma}$ and $\mathcal{I}_{\varsigma^{\prime}}$ coincide.

Let us recall that quantum Poisson algebras $(\mathrm{qPa})$ canonically induce classical Poisson algebras $(\mathrm{cPa})$ in the sense of $[15]$. We denote by $\mathcal{S}(\mathcal{D})$ the cPa implemented by a qPa $\mathcal{D}$. We also know that $\mathcal{S}(\mathcal{D}(M))$ coincides with the algebra $\operatorname{Sec}(\mathcal{S} T M)$ of symmetric contravariant tensor fields over $M$ and with the algebra $\operatorname{Pol}\left(T^{*} M\right)$ of smooth functions on the cotangent bundle of $M$ that are polynomial along the fibers.

TheOrem 2. The classical Poisson algebras $\mathcal{S}(\mathcal{D}(L))$ and $\mathcal{S}(\mathcal{D}(M))$ induced by $\mathcal{D}(L)$ and $\mathcal{D}(M)$ are canonically isomorphic cPa.

Proof. In any qPa $\mathcal{D}$ we can define the $k$ th-order symbol $\sigma_{k}(D)$ of any differential operator $D \in \mathcal{D}$, the degree of which is $\leq k$ (see [15]). In the fundamental case $\mathcal{D}=\mathcal{D}(M)$, this algebraically defined symbol coincides with the usual geometric $k$ th-order symbol. It is then clear (see (12)) that

$$
\sigma_{k}\left(\mathcal{I}_{\varsigma}(\Delta)\right)=\sigma_{k}\left(\mathcal{I}_{\varsigma^{\prime}}(\Delta)\right), \quad \forall \Delta \in \mathcal{D}^{k}\left(L_{U}\right)
$$

It is obvious that $\sigma_{k}\left(\mathcal{I}_{\varsigma}(\Delta)\right) \in \mathcal{S}_{k}(\mathcal{D}(U)), \Delta \in \mathcal{D}^{k}\left(L_{U}\right)$ only depends on the $k$ th-order symbol of $\Delta$, i.e. is actually defined on $\mathcal{S}_{k}\left(\mathcal{D}\left(L_{U}\right)\right)$. Indeed, for any $P \in \mathcal{S}_{k}\left(\mathcal{D}\left(L_{U}\right)\right)$, if $P=\sigma_{k}(\Delta)=\sigma_{k}\left(\Delta^{\prime}\right)$, we have $\sigma_{k}\left(\mathcal{I}_{\varsigma}(\Delta)\right)-\sigma_{k}\left(\mathcal{I}_{\varsigma}\left(\Delta^{\prime}\right)\right)=0$, since $\sigma_{k}\left(\Delta-\Delta^{\prime}\right)=0$. So

$$
\Phi_{\varsigma}^{U}: \mathcal{S}_{k}\left(\mathcal{D}\left(L_{U}\right)\right) \ni P \rightarrow \sigma_{k}\left(\mathcal{I}_{\varsigma}\left(\sigma_{k}^{-1}(P)\right)\right) \in \mathcal{S}_{k}(\mathcal{D}(U))
$$

is a $\mathrm{cPa}$ isomorphism. The morphism properties with respect to the associative commutative and the Poisson-Lie multiplications are direct consequences of the definitions of these operations . and $\{.,$.$\} :$

$$
\begin{gathered}
\sigma(\Delta) \cdot \sigma\left(\Delta^{\prime}\right)=\sigma_{\operatorname{deg} \Delta+\operatorname{deg} \Delta^{\prime}}\left(\Delta \circ \Delta^{\prime}\right), \\
\left\{\sigma(\Delta), \sigma\left(\Delta^{\prime}\right)\right\}=\sigma_{\operatorname{deg} \Delta+\operatorname{deg} \Delta^{\prime}-1}\left(\left[\Delta, \Delta^{\prime}\right]\right),
\end{gathered}
$$


where $\sigma(\Delta)$ and $\operatorname{deg} \Delta$ are the principal symbol and the degree of $\Delta$ respectively (see $[15])$. In view of (13) the isomorphisms $\Phi_{\varsigma}^{U}$ define a global $\mathrm{cPa}$ isomorphism

$$
\Phi: \mathcal{S}(\mathcal{D}(L)) \rightarrow \mathcal{S}(\mathcal{D}(M))
$$

such that

$$
\left.(\Phi P)\right|_{U}=\Phi_{\varsigma}^{U}\left(\left.P\right|_{U}\right)
$$

for any $P \in \mathcal{S}_{k}(\mathcal{D}(L))$ and a trivialization $\varsigma$ of $L$ over a member $U$ of some appropriate open covering of $M$. Let us mention that if $P=\sigma_{k}(D), D \in \mathcal{D}^{k}(L)$, the restriction $\left.P\right|_{U}=\left.\left(\sigma_{k}(D)\right)\right|_{U}$ is nothing but the well-defined class $\sigma_{k}\left(\left.D\right|_{U}\right)$ of the restriction to $U$ of the local operator $D$.

Theorem 3. The quantum Poisson algebras $\mathcal{D}(L)$ and $\mathcal{D}(M)$ are isomorphic.

Proof. Let $L_{0}=L \backslash\{0\}$ be the bundle $L$ with removed 0 -section and let $\left|L_{0}\right|=L_{0} / \mathbb{Z}_{2}$ be the quotient of $L_{0}$ with respect to the obvious action of the multiplicative group $\mathbb{Z}_{2}=\{-1,1\}$. This quotient $\left|L_{0}\right|$ is an affine bundle of dimension 1 (i.e. a smooth bundle of 1-dimensional affine spaces, such that the passage from one trivialization to another is given by an affine map) canonically modelled on the vector bundle $M \times \mathbb{R}$. Indeed, on the fibers of $\left|L_{0}\right|$ there is a canonical free and transitive action of $\mathbb{R}$ induced by the Liouville vector field of $L$ which projects onto a nowhere-vanishing vector field on $\left|L_{0}\right|$. This action turns $\left|L_{0}\right|$ into an $\mathbb{R}$-principal bundle. Since the fibers are contractible, $\left|L_{0}\right|$ has a global section $|\eta|$. If $\left(U_{\alpha}\right)_{\alpha \in \Lambda}$ is a covering of $M$ by open connected subsets over which $L$ is trivializable, the section $|\eta|$ can be viewed as a family $\left(\left\{\eta_{\alpha},-\eta_{\alpha}\right\}\right)_{\alpha \in \Lambda}$ of pairs of non-vanishing local sections of $L$ such that $\left\{\eta_{\alpha},-\eta_{\alpha}\right\}=\left\{\eta_{\beta},-\eta_{\beta}\right\}$ on $U_{\alpha} \cap U_{\beta}$. This follows immediately from the above depicted trivializations. When choosing for each $\alpha$ a representative $\tilde{\eta}_{\alpha} \in\left\{\eta_{\alpha},-\eta_{\alpha}\right\}$, we get a family of nowhere vanishing local sections of $L$, such that on $U_{\alpha} \cap U_{\beta}$, we have $\tilde{\eta}_{\alpha}= \pm \tilde{\eta}_{\beta}$ that reflects the fact that line bundles over $M$ are classified by $H^{1}\left(M ; \mathbb{Z}_{2}\right)$. In view of (11) and (12) we then get a global quantum Poisson algebra isomorphism between $\mathcal{D}(L)$ and $\mathcal{D}(M)$.

The preceding result shows that the Lie algebra $\mathcal{D}(L \rightarrow M)$ characterizes the smooth structure of the base manifold $M$, but does not recognize the topological complications in $L$.

Corollary 1. Let $\pi: L \rightarrow M$ and $\pi^{\prime}: L^{\prime} \rightarrow M^{\prime}$ be two real vector bundles of rank 1 over two smooth manifolds $M$ and $M^{\prime}$ respectively. The Lie algebras $\mathcal{D}(L \rightarrow M)$ and $\mathcal{D}\left(L^{\prime} \rightarrow\right.$ $\left.M^{\prime}\right)$ are isomorphic if and only if the base manifolds $M$ and $M^{\prime}$ are diffeomorphic.

Proof. Immediate consequence of Theorem 3 and of [15, Theo. 6].

Note that the essential fact is that $\mathcal{D}(L \rightarrow M)$ and $\mathcal{D}\left(L^{\prime} \rightarrow M\right)$ are always isomorphic (even as qPa), whether or not $L$ and $L^{\prime}$ are isomorphic as vector bundles. However these isomorphisms are not canonical, depending on the choice of the section $|\eta|$ of $\left|L_{0}\right|$. This observation, together with the description of automorphisms and derivations of $\mathcal{D}(M)$ and $\mathcal{D}^{1}(M)[15,17]$, gives automatically the obvious description of automorphisms and derivations of $\mathcal{D}(L \rightarrow M)$ and $\mathcal{D}^{1}(L \rightarrow M)$. The only difference is that diffeomorphims $\phi$ of $M$ (resp. vector fields on $M$ ) do not define automorphisms (resp. derivations) of 
$\mathcal{D}(L \rightarrow M)$ and $\mathcal{D}^{1}(L \rightarrow M)$ canonically, but in the way depending on the choice of the section $|\eta|$ of $\left|L_{0}\right|$. For example, the derivation $C_{X}^{|\eta|}$ associated with a vector field $X$ on $M$ and a choice of $|\eta|$ is defined locally by

$$
C_{X}^{|\eta|}(D)\left(f \eta_{\alpha}\right)=\left[X\left(D_{\alpha}(f)\right)-D_{\alpha}(X(f))\right] \eta_{\alpha},
$$

where $D_{\alpha}(f) \eta_{\alpha}=D\left(f \eta_{\alpha}\right)$. This definition is correct, since nothing changes when we choose $-\eta_{\alpha}$ instead of $\eta_{\alpha}$.

\section{References}

[1] K. Abe, Pursell-Shanks type theorem for orbit spaces and G-manifolds, Publ. Res. Inst. Math. Sci. 18 (1982), 265-282.

[2] I. Amemiya, Lie algebra of vector fields and complex structure, J. Math. Soc. Japan 27 (1975), 545-549.

[3] C. J. Atkin and J. Grabowski, Homomorphisms of the Lie algebras associated with a symplectic manifold, Compos. Math. 76 (1990), 315-348.

[4] A. Avez, A. Diaz-Miranda and A. Lichnerowicz, Sur l'algèbre des automorphismes infinitésimaux d'une variété symplectique, J. Diff. Geom. 9 (1974), 1-40.

[5] M. De Wilde and P. Lecomte, Some characterizations of differential operators on vector bundles, in: E. B. Christoffel et al. (eds.), Birkhäuser Verlag, Basel 1981, 543-549.

[6] M. De Wilde and P. Lecomte, Cohomology of the Lie algebra of smooth vector fields of a manifold, associated to the Lie derivative of smooth forms, J. Pure Appl. Math. (9) 62, no 2 (1983), 197-214.

[7] P. A. M. Dirac, The Principles of Quantum Mechanics, Chap. IV, Oxford University Press, Oxford 1958.

[8] D. B. Fuchs, Cohomology of Infinite-Dimensional Lie Algebras, Consultants Bureau, New York, 1987.

[9] M. Gotay and J. Grabowski, On quantizing nilpotent and solvable basic algebras, Canadian Math. Bull. 44 (2001), 140-149.

[10] J. Grabowski, Isomorphisms and ideals of the Lie algebras of vector fields, Invent. Math. 50 (1978), 13-33.

[11] J. Grabowski, Derivations of the Lie algebras of analytic vector fields, Compositio Math. 43 (1981), 239-252.

[12] J. Grabowski, Derivations of the Lie algebras associated with a symplectic structure, in: Differential Geometry and Its Applications (Brno, 1986), D. Krupka and A. Švec (eds.), $117-125$.

[13] J. Grabowski, Free subgroups of diffeomorphism groups, Fund. Math. 131 (1988), 103-121.

[14] J. Grabowski, Lie algebras of vector fields and generalized foliations, Publ. Mat. 37 (1993), 359-367.

[15] J. Grabowski and N. Poncin, Automorphisms of quantum and classical Poisson algebras, Comp. Math. 140 (2004), 511-527.

[16] J. Grabowski and N. Poncin, Lie-algebraic characterizations of manifolds, Cent. Eur. J. Math. 2 (2005), 811-825.

[17] J. Grabowski and N. Poncin, Derivations of the Lie algebras of differential operators, Indag. Math. N.S. 16 (2005), 181-200. 
[18] J. Grabowski, G. Marmo and P. W. Michor, Homology and modular classes of Lie algebroids, Ann. Inst. Fourier. 56 (2006), 69-83.

[19] H. Hauser and G. Müller, Affine varieties and Lie algebras of vector fields, Manusc. Math. 80 (1993), 309-337.

[20] A. Joseph, Derivations of Lie brackets and canonical quantization, Comm. Math. Phys. 17 (1970), 210-232.

[21] Y. Kanie, Cohomologies of Lie algebras of vector fields with coefficients in adjoint representations. Case of classical type, Publ. Res. Inst. Math. Sci. 11 (1975), 213-245.

[22] Y. Kanie, Cohomologies of Lie algebras of vector fields with coefficients in adjoint representations. Foliated case, Publ. Res. Inst. Math. Sci. 14 (1978), 487-501.

[23] Y. Kanie, Some Lie algebras of vector fields on foliated manifolds and their derivation algebras, Proc. Japan Acad. Ser. A Math. Sci. 55 (1979), 409-411.

[24] Y. Kanie, Some Lie algebras of vector fields on foliated manifolds and their derivations: Case of partially classical type, Nagoya Math. J. 82 (1981), 175-207.

[25] N. Kopell, Commuting diffeomorphisms, in: Global Analysis (Berkeley, CA, 1968), Proc. Sympos. Pure Math. 14, Amer. Math. Soc., Providence, R.I., 1970, 165-184.

[26] P. Lecomte, Sur les champs de densités sur une variété, preprint, University of Liège 2003 (electronic version at http://www.ulg.ac.be/geothalg/QPE/index.html).

[27] H. Omori, Infinite Dimensional Lie Transformation Groups, Lect. Notes in Math. 427, Springer-Verlag, 1976.

[28] M. E. Shanks and L. E. Pursell, The Lie algebra of a smooth manifold, Proc. Amer. Math. Soc. 5 (1954), 468-472.

[29] S. M. Skryabin, The regular Lie rings of derivations of commutative rings, preprint VINITI 4403-V87, Moscow University, 1987.

[30] F. Takens, Derivation of vector fields, Comp. Math. 26 (1973), 151-158.

[31] L. S. Wollenberg, Derivations of the Lie algebra of polynomials under Poisson bracket, Proc. Amer. Math. Soc. 20 (1969), 315-320. 